\title{
Stability and fairness in the job scheduling problem
}

\author{
Eric Bahel (Virginia Tech) \\ Christian Trudeau (University of Windsor)
}

Working paper 18 - 03

Working papers are in draft form. This working paper is distributed for purposes of comment and discussion only. It may not be reproduced without permission of the copyright holder. Copies of working papers are available from the author or at http://ideas.repec.org/s/wis/wpaper.html. 


\title{
Stability and fairness in the job scheduling problem*
}

\author{
Eric Bahel ${ }^{\dagger} \quad$ Christian Trudeau ${ }^{\ddagger}$
}

May 3, 2018

\begin{abstract}
The job scheduling problem is a classic operational research problem in which agents have jobs to be executed by machines in given time slots, with each machine being able to process only one job at a time. We study this problem using cooperative game theory, focusing on how to divide the minimum cost (of executing all jobs) between the agents. First, we characterize the set of stable allocations, which all charge only users whose jobs are executed in peak-demand time periods. Second, using properties designed to avoid strategic mergers or splits of the jobs, we offer axiomatizations for two remarkable stable allocation rules. Third, observing that all stable rules fail Unanimity Lower Bound (ULB), a property requiring that everybody pay an equal share of the first machine (since it is needed by all), we study and axiomatize the Shapley value, which satisfies ULB. A compromise is then proposed between Stability and ULB.
\end{abstract}

JEL classification numbers: C71, D63

Keywords: game theory, cost sharing, job scheduling, stability, unanimity lower bound, Shapley value.

\footnotetext{
* Christian Trudeau acknowledges financial support by the Social Sciences and Humanities Research Council of Canada [grant number 435-2014-0140]. Authors thank Hervé Moulin, Juan Morero-Ternero and participants in the "Advances in Fair Division" conference (St. Petersburg, August 2017) for helpful dicussions. Esaignani Selvarajah provided helpful assistance on the operations research problem.

${ }^{\dagger}$ Department of Economics, Virginia Polytechnic Institute and State University, Blacksburg, VA 24061, USA. Email: erbahel@vt.edu

${ }^{\ddagger}$ Corresponding author. Tel: 1-519-253-3000 ext. 2374. Department of Economics, University of Windsor, 401 Sunset Avenue, Windsor, Ontario, Canada. Email: trudeauc@uwindsor.ca
} 


\section{Introduction}

Consider a group of agents who each have to execute a number of jobs performed by machines. These jobs may differ in their lengths, starting and ending times. A machine can only execute one job at a time, and the necessity to execute multiple jobs (with potential time conflicts) thus creates a "job scheduling problem". As a prime illustration of this problem, consider the school bus system in Ontario, Canada. In most major cities, four school districts compete for students and each district hires school buses to pick up its kids. Schedules vary from one school to the other, allowing for a bus and its driver to cover routes for at least two different districts. The consortium of bus companies then charges each school district for its use of the buses. Other examples include scheduling classes in classrooms, assigning planes to mechanics, with planes having a short window for maintenance between landing and take-off, and assigning cases to lawyers, while preventing conflicts of interest that would occur if cases overlapped.

The job scheduling problem is a classic topic in operations research. There exist many variants of this problem; and our version is equivalent to the Tactical Fixed Job Scheduling problem, as defined in the review of Elliyi and Azizgoglu (2004). Early examples include Dantzig and Fulkerson (1954), who study the minimum number of tankers to carry on a schedule of supply jobs, Gertsbakh and Stern (1978), who link the problem to others, and propose an extension by considering variable starting times. Closest to our model is the setting of Gupta et al. (1979): given a set of time intervals, the authors look at the problem of finding a minimal partition such that no element of the partition contains two overlapping intervals. They find a $\mathrm{T}(\mathrm{N} \log \mathrm{N})$ algorithm which is optimal.

A number of extensions (different costs for different machines, machines available at different times, different types of jobs and machines only able to complete jobs of a given type, etc.) have been proposed. See Elliyi and Azizgoglu (2004) for a review.

To our knowledge, the job scheduling problem has not been studied in economics, although it is related to the assignment problem (Shapley and Shubik (1971)). If a coalition $S$ is compatible (i.e., the jobs of all members of $S$ can be done on the same machine), they create a value equivalent to the cost of $|S|-1$ machines. The closest problem seems to be the glove market game (Shapley, 1959). Our model is different because agents are not assigned to a given side of the market, which is typically partitioned into elements of different sizes.

Another similar problem is the Bohm-Bawerk horse market game in which agents value all goods in the same manner, but reserve prices might differ accross agents. See for instance Núñez and Rafels (2005) and Tejada (2013), who study respectively the core of two-sided and multi-sided Bohm-Bawerk games.

Conceptually, the problem is closely related to the queuing problem, in which we also have to assign agents to machines. The main difference with the job scheduling problem is that jobs do not need to be executed at a precise time. Instead, each agent has a waiting cost, and the objective is to assign all jobs to a single machine, while minimizing aggregate waiting costs. See Chun (2016) for a 
review. The differences are about the rigidities in the models: fair queuing limits the number of machines (typically to one), but jobs can be moved around (which is determined within the problem), while job scheduling allows for multiple machines (with the number determined optimally), but there is no flexibility for the starting times of the jobs.

After describing the operations research problem and its associated cooperative game in Section 2, we examine the core in Section 3. We find that for an allocation to be in the core, it must charge only the so-called peak-demand coalitions, i.e., groups of pairwise-incompatible players that need the same number of machines as the grand coalition to execute their jobs. We axiomatize two cost sharing rules that produce a core allocation for each problem. The first one charges the same amount to all peak-demand coalitions. The second one assigns to each peak-demand coalition a cost share that is proportional to the length of its job interval. The characterizations that we propose for these rules are based on properties that prevent strategic merging or splitting of jobs, in the spirit of axioms proposed by O'Neill (1992), Chun (1988) and Sprumont (2005) in various contexts.

Since all coalitions need at least one machine to process their jobs, it seems very natural to impose that each agent pay at least $\frac{1}{n} t h$ of the cost of a machine, a property that we call Unanimity Lower Bound, following the propositions of Moulin (1990), Maniquet (1996) and Hougaard and Moulin (2014) in different contexts. However, this fairness requirement is incompatible with Core Selection. We examine in Section 4 the Shapley value, which fails Core Selection but satisfies Unanimity Lower Bound. The axiomatic analysis that we propose is based on the property of Equal treatment: if two agents become incompatible (with all else equal), they should see their cost shares affected in the same manner. The property is inspired by Myerson (1977) and Kar (2002).

In Section 5, we propose a compromise between Core Selection and Unanimity Lower Bound, by weakening Core Selection: we suppose that if a coalition wants to quit the group, it will be held responsible for its fair share of the "fixed cost", which in our case is the cost of the first machine. We propose modifications to the rules introduced in Section 3 that satisfy the Weak Core Selection property and Unanimity Lower Bound.

Finally, in Section 6, we drop the assumption that all machines have the same cost. We show that the core is non-empty if and only if the average cost of a machine decreases with the number of machines used. Section 7 concludes and discusses further extensions. Independence of the properties used in the axiomatizations is shown in the Appendix.

\section{Preliminaries}

\section{$2.1 \quad$ The model}

Let $N=\{1, \ldots, n\}$ be a finite set of agents, with $n \geq 2$. We denote by $\mathcal{N}$ the collection of all such sets $N$. Each agent $i \in N$ has a single job to be executed; 
andher job is described by its starting time and finishing time, respectively, $s_{i}$ and $f_{i}$, with $0 \leq s_{i}<f_{i} \leq 1$. Every agent is thus characterized by a pair $\left(s_{i}, f_{i}\right)$; and we write $J=\left\{\left(s_{i}, f_{i}\right)\right\}_{i \in N}$ to refer to the agents' job profile. Let $\mathcal{J}(N)$ be the set of all such job profiles (listing the jobs of members of $N$ ).

A job scheduling problem can then be defined as a pair $(N, J)$ such that $N=\{1,2, \ldots, n\} \in \mathcal{N}$ and $J \in \mathcal{J}(N)$. We call $\mathcal{D}$ the set containing all such job scheduling problems.

Each job must be processed on a machine; and each machine is able to perform only one job at a time, without interruption from starting time to finishing time. Assume that all machines have the same cost, which is normalized to 1 without loss of generality; and the full cost of 1 must be paid for each machine that is used (for any positive amount of time).

For all $S \subseteq N, J \in \mathcal{J}(N)$ and $t \in[0,1]$, define $C(S, J, t)=\left\{i \in S \mid s_{i} \leq t<f_{i}\right\}$. That is to say, $C(S, J, t)$ is the set of users (within $S$ ) whose jobs are ongoing at time $t$. In addition, we write $c(S, J, t)=|C(S, J, t)|$; and we define the time intersection of the jobs of $S \subseteq N$ as $I(S) \equiv\left[\max _{i \in S} s_{i}, \min _{i \in S} f_{i}\right)=\cap_{i \in S}\left[s_{i}, f_{i}\right)$.

Definition 1 Consider $N=\{1,2, \ldots, n\}$ and $J \in \mathcal{J}(N)$.

(a)-Denote by $\bar{c}^{J}(S) \equiv \max _{t \in[0,1]} c(S, J, t)$ the peak demand for each coalition $S \subset N$, that is, the highest number of users that we have at the same time.

(b)- Call a (nonempty) subset of agents $S \in 2^{N}$ a compatible coalition if $\bar{c}^{J}(S)=1$, that is, if their jobs can all be executed on the same machine.

We further define the intersection of jobs of agents in $S \subseteq N$ as $I(S) \equiv$ $\left[\max _{i \in S} s_{i}, \min _{i \in S} f_{i}\right)=\cap_{i \in S}\left[s_{i}, f_{i}\right)$.

For a problem $(N, J)$, a feasible production plan is a partition $P=\left\{P^{1}, \ldots, P^{K}\right\}$ of the set $N$ such that each $P^{k}(k=1, \ldots, K)$ is a compatible coalition. Given a problem $(N, J)$, an optimal production plan for a coalition is a feasible production plan that minimizes cost of completing all jobs listed in $J$. Since the cost of using any machine is 1, optimality amounts to using as few machines as possible to execute all jobs. In other words, an optimal production plan is a partition of $N$ into the least possible number of compatible coalitions.

Obviously, an optimal production plan $P=\left\{P^{1}, \ldots, P^{K}\right\}$ must satisfy $K \geq$ $\bar{c}^{J}(N)$. Interestingly, it has been shown that there always exists a feasible production plan such that $K=\bar{c}^{J}(N)$.

Lemma 1 (Kroon et al., 1995)

Consider a fixed problem $(N, J)$. There always exists a feasible production plan $\left\{P^{1}, \ldots, P^{K}\right\}$ such that $K=\bar{c}^{J}(N)$.

Such a production plan can be found using the following algorithm. First, assign to the first machine the agent whose job starts the earliest. Keep adding to the first machine the agent with the earliest starting job compatible with previously added jobs until no more jobs are compatible. Then, repeat the process for additional machines, as needed. Let us use the following example to illustrate the previous definitions and lemma. 
Example 1 Suppose that $N=\{1,2,3,4,5\}$ with the jobs described by $J=$ $\{(0,0.1),(0.2,0.4),(0.4,0.6),(0.3,0.5),(0.5,0.7)\}$. This problem is illustrated by Figure 1. One can easily see that $c(N, J, t)=2$ for $t \in[0.3,0.7)$ and

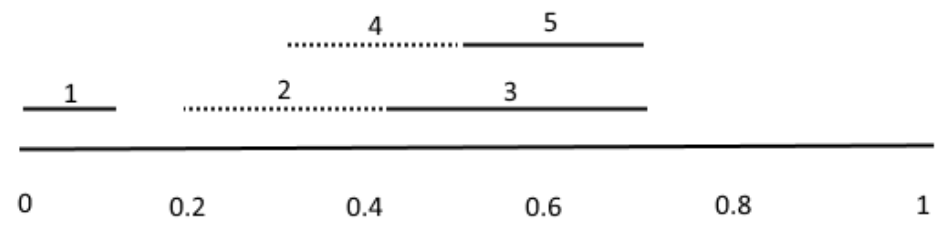

Figure 1: Example of a job scheduling problem

$c(N, J, t) \leq 1$ otherwise. Thus, $\bar{c}^{J}(N)=2$. Optimal production plans are $P^{1}=\{\{1,2,3\},\{4,5\}\}$ and $P^{2}=\{\{1,4,5\},\{2,3\}\}$. In particular, if we apply the described algorithm to our example, then the jobs 1, 2 and 3 (which are compatible) are assigned to the first machine, and jobs 4 and 5 are assigned to the second machine.

Given a problem $(N, J)$, recall from what precedes that $\bar{c}^{J}(S)$ is the cost of completing all jobs in $S$. Using the convention, $\bar{c}^{J}(\emptyset)=0$, one can easily see that $\bar{c}^{J}(\cdot)$ is the characteristic cost function of the coalitional game generated by the job scheduling problem $(N, J)$.

An allocation $y \in \mathbb{R}_{+}^{N}$ is a distribution of the minimum cost of serving $N$ between its agents so as to satisfy the budget balance condition: $\sum_{i \in N} y_{i}=\bar{c}^{J}(N)$. We write $y_{S} \equiv \sum_{i \in S} y_{i}$. A cost sharing rule $y$ assigns an allocation $y(N, J)$ to every job scheduling problem $(N, J)$, which we formalize in the following definition.

Definition 2 A cost sharing rule is a mapping $y: \mathcal{D} \rightarrow \mathbb{R}^{N}$ such that $\sum_{i \in N} y_{i}(N, J)=$ $\bar{c}^{J}(N)$, for any $(N, J) \in \mathcal{D}$.

We are now able to state the following definition.

Definition 3 The core of the problem $(N, J)$ is Core $(N, J)=\left\{y \in \mathbb{R}^{N} \mid y_{N}=\right.$ $\left.\bar{c}^{J}(N), y_{S} \leq \bar{c}^{J}(S), \forall S \subset N\right\}$.

That is to say, the core of $(N, J)$ is the set of allocations such that no coalition $S$ jointly pays more than its stand-alone cost $\bar{c}^{J}(S)$. Picking a core allocation is a central requirement in cooperative game theory: any allocation not in the core will be blocked by at least one coalition of agents. The preliminary result below is straightforward.

Lemma 2 Consider a fixed problem $(N, J)$ and let $\left\{P^{1}, \ldots, P^{K}\right\}$ be an efficient production plan for $(N, J)$. If $y \in \operatorname{Core}(N, J)$ then we have $y_{P^{k}}=1$, for any $k=1, \ldots, K$. 
The proof is omitted; it follows directly from the respective definitions of the core and an efficient allocation of the production. In the following section, we provide a characterization of the core for a job scheduling problem.

\section{Core allocations: the peak-demand and peak- interval rules}

A natural way of studying this problem is through the lens of the stand-alone core. We thus first describe the core allocations, before introducing two particular cost-sharing rules which always propose an allocation that is in the core. We then provide an axiomatic characterization for each of these two rules.

\subsection{Study of the core}

Let us fix a problem $(N, J)$. We say that a coalition $S$ is a peak-demanding coalition if the group requires the same number of machines as the grand coalition and if none of its members are compatible. Formally, let

$$
G^{P D}=\left\{S \subseteq N\left|\bar{c}^{J}(S)=\bar{c}^{J}(N)=\right| S \mid\right\} ; \text { and write } \bar{g}=\left|G^{P D}\right| .
$$

For each $i \in N$, call $G_{i}^{P D}=\left\{S \subseteq G^{P D} \mid i \in S\right\}$ the set of all peak-demanding coalitions that $i$ belongs to, and let $g_{i}^{P D}=\left|G_{i}^{P D}\right|$ be its cardinality.

Consider a (normalized) weight function $w \in \mathbb{R}_{+}^{G^{P D}}$ satisfying $\sum_{T \in G^{P D}} w_{T}=$ 1 ; and then define the associated cost share: for any $i \in N$

$$
y_{i}^{w}=\left\{\begin{array}{cl}
\sum_{S \in G_{i}^{P D}} w_{S}, & \text { if } G_{i}^{P D} \neq \emptyset \\
0, & \text { otherwise. }
\end{array}\right.
$$

We call $W(N, J)$ the set containing all such weight functions $w \in \mathbb{R}_{+}^{G^{P D}}$ for the problem $(N, J)$; and we use the notation $\mathcal{Y}(N, J) \equiv\left\{y^{w} \mid w \in W(N, J)\right\}$ for the set containing the allocations generated via (2) for all possible weight functions.

Example 2 Let us revisit the job scheduling problem of Example 1. We have $G^{P D}=\{\{2,4\},\{3,4\},\{3,5\}\}$. Pick any $w_{\{2,4\}}, w_{\{3,4\}}, w_{\{3,5\}} \geq 0$ such that $w_{\{2,4\}}+w_{\{3,4\}}+w_{\{3,5\}}=1$. One then obtains cost allocations of the form $y^{w}=\left(0, w_{\{2,4\}}, w_{\{3,4\}}+w_{\{3,5\}}, w_{\{2,4\}}+w_{\{3,4\}}, w_{\{3,5\}}\right)$. It is easy to verify that $y^{w}$ is in the core of this particular problem. In fact, it can be shown that the core of this problem contains only allocations of this type.

The result of Example 2 is not a coincidence. The following theorem, which is one of our main results, provides a full characterization of the core of a job scheduling problem. It states precisely that the core coincides with the set of allocations $\mathcal{Y}(N, J)$ defined earlier. 
Theorem 1 For any problem $(N, J) \in \mathcal{D}$, we have Core $(N, J)=\mathcal{Y}(N, J)$.

Proof. Considering a fixed problem $(N, J) \in \mathcal{D}$, we have to show the two inclusions $\mathcal{Y}(N, J) \subseteq \operatorname{Core}(N, J)$ and $\operatorname{Core}(N, J) \subseteq \mathcal{Y}(N, J)$.

The first inclusion is relatively easy to prove. Indeed, for any given peakdemand coalition $S \in G^{P D}$, define the allocation $y^{S}$ by $y_{i}^{S}= \begin{cases}1, & \text { if } i \in S ; \\ 0, & \text { otherwise. }\end{cases}$

It is straightforward to check that $y^{S}$ is stable [since every coalition $T$ pays a joint share of $\left.y_{T}^{S}=|T \cap S| \leq \bar{c}^{J}(T)\right]$. We thus have the desired result since the core is a convex set and $W(N, J)$ is the convex hull of the set of allocations $\left\{y^{S}, S \in G^{P D}\right\} \subset \operatorname{Core}(N, J)$.

To prove the second inclusion, let us first remark from Definition 1-(a) and Equation (1) that each coalition $S \in G^{P D}$ is associated with a unique nonempty interval $I(S)=\left[\max _{i \in S} s_{i}, \min _{i \in S} f_{i}\right)=\cap_{i \in S}\left[s_{i}, f_{i}\right)$. Furthermore, if $S, S^{\prime} \in G^{P D}$ and $S \neq S^{\prime}$, then we have $I(S) \cap I\left(S^{\prime}\right)=\emptyset$. This allows us to rank the respective peak-demand coalitions $S_{1}, \ldots, S_{\bar{g}}$, with the associated time intervals $I_{1}, \ldots, I_{\bar{g}}$ such that $t<t^{\prime}$ whenever $\left(t, t^{\prime}\right) \in I_{g} \times I_{g+1}$ and $g \in\{1, \ldots, \bar{g}-1\}$. In addition, for all $g=1, \ldots, \bar{g}$, we write $i_{g}$ to refer to (one of) the agent(s) whose finishing time is earliest in $S_{g}$, that is, $f_{i_{g}}=\min _{i \in S_{g}} f_{i}$.

Considering an arbitrary stable allocation $y \in \operatorname{Core}(N, J)$, we will construct a vector of weights $w \in \mathbb{R}_{+}^{G^{P D}}$ and show that $y=y^{w}$. First, let $w_{1}=y_{i_{1}}$ and, if $\bar{g} \geq 2$, define recursively $w_{g}=y_{i_{g}}-\sum_{\substack{l<g \\ i_{g} \in S_{l}}} w_{l}$ for $g=2, \ldots, \bar{g}$.

Fix an efficient allocation of the production $P=\left\{P^{1}, \ldots, P^{K}\right\}$ for the problem $(N, J)$, that is, $K=\bar{c}^{J}(N)$ is the minimum number of machines to execute the jobs $J$. For any $i \in N$, call $k_{i}$ the rank of the unique subset of the partition $P$ such that $i \in P^{k_{i}}$. Without loss of generality, rank the $n$ agents in (weakly) increasing order of finishing time, that is, $f_{1} \leq f_{2} \leq \ldots \leq f_{n}$. We use induction over this order of finishing time to prove that $y=y^{w}$.

Step 1: Note from what precedes that we necessarily have $f_{1} \leq f_{i_{1}}$, with $\overline{G_{1}^{P D}}=\emptyset$ if $f_{1}<f_{i_{1}}$. Let us then show that $y_{1}= \begin{cases}0, & \text { if } f_{1}<f_{i_{1}} \\ w_{1}, & \text { if } f_{1}=f_{i_{1}} .\end{cases}$

Indeed, assuming that $f_{1}<f_{i_{1}}$ and recalling then that $G_{1}^{P D}=\emptyset$, we can claim that there exists $\bar{k} \in\{1, \ldots, K\} \backslash\left\{k_{1}\right\}$ such that $\lim _{t \rightarrow f_{1}} C\left(P_{\bar{k}}, J, t\right)=\emptyset$. In words, since the job of agent 1 is not in a peak demand interval, we can find a machine of the partition $P$ that is free at any time $t$ that is close enough to $f_{1}$. But since we have by definition $f_{1} \leq f_{j}$ for any $j \in N$, we can claim that $C\left(P_{\bar{k}}, J, t\right)=\emptyset, \forall t \in\left[s_{1}, f_{1}\right)$, that is to say, the job of agent 1 is compatible with every job in $P_{\bar{k}}$. Hence, we have found a new efficient allocation of the production $P^{\prime}=\left\{P^{\prime 1}, \ldots, P^{\prime K}\right\}$, defined by: $P^{\prime k}=P^{k}$ if $k \notin\left\{k_{1}, \bar{k}\right\} ; P^{\prime k_{1}}=$ $P^{k_{1}} \backslash\{1\}$; and $P^{\prime k}=P^{\bar{k}} \cup\{1\}$. It thus follows from Lemma 2 (applied to both $P$ and $\left.P^{\prime}\right)$ that $y_{P^{\bar{k}}}+y_{1}=1=y_{P_{\bar{k}}}$ and, therefore, $y_{1}=0$.

Suppose now that we instead have $f_{1}=f_{i_{1}}$. Then we have two possibilities: $i_{1}=1$ or $\left(i_{1} \neq 1\right.$ and $\left.f_{1}=f_{i_{1}}\right)$. The case where $i_{1}=1$ is trivial since we have 
by definition $y_{1}=y_{i_{1}}=w_{1}$. Assume then that $i_{1} \neq 1$ and $f_{1}=f_{i_{1}}$. Then one can see that we have a new efficient allocation of the production in $P^{\prime \prime}=$ $\left\{P^{\prime \prime 1}, \ldots, P^{\prime \prime K}\right\}$ such that: $P^{\prime \prime k}=P^{k}$ if $k \notin\left\{k_{1}, k_{i_{1}}\right\} ; P^{\prime \prime k_{1}}=\left(P^{k_{1}} \cup\left\{i_{1}\right\}\right) \backslash\{1\}$; and $P^{\prime \prime k_{i_{1}}}=\left(P^{k_{i_{1}}} \cup\{1\}\right) \backslash\left\{i_{1}\right\}$. Indeed, since $i_{1} \neq 1$ and $f_{1}=f_{i_{1}}=\min _{j \in N} f_{j}$, we can claim that $\left(P^{k_{1}} \cup\left\{i_{1}\right\}\right) \backslash\{1\}$ and $\left(P^{k_{i_{1}}} \cup\{1\}\right) \backslash\left\{i_{1}\right\}$ are compatible coalitions (with an empty intersection). Applying Lemma 2 to $P$ and $P^{\prime \prime}$ thus gives: $y_{\left(P^{k_{1}} \cup\left\{i_{1}\right\}\right) \backslash\{1\}}=y_{P^{k_{1}}}+y_{i_{1}}-y_{1}=1=y_{P^{k_{1}}}$. It hence comes that $y_{1}=y_{i_{1}}=w_{1}$. In summary, we have shown that $y_{1}$ is given by Equation (2).

Step $i \geq 2$ : Suppose now that for some $i=2, \ldots, n$, we have $f_{i-1} \leq f_{i}$ [with $\left.\overline{f_{j} \notin\left(f_{i-1}\right.}, f_{i}\right)$ for any $\left.j=1, \ldots, n\right]$; and by our induction hypothesis, assume that for any $j=1, \ldots, i-1$ we have

$$
y_{j}=y_{j}^{w}=\left\{\begin{array}{cl}
0, & \text { if } G_{j}^{P D}=\emptyset ; \\
\sum_{S_{g} \in G_{j}^{P D}} w_{g} & \text { otherwise. }
\end{array}\right.
$$

We will now show that $y_{i}=y_{i}^{w}$ also holds by distinguishing the two cases below.

Case 1: Suppose that $f_{i} \neq f_{i_{g}}$ for any $g=1, \ldots, \bar{g}$.

Then we have $[\underbrace{\left[f_{i-1}, f_{i}\right]}_{\neq \emptyset} \cap I_{g}=\emptyset$ (for any $g=1, \ldots \bar{g}$ ) and the following thus holds: $\exists \bar{k} \in\{1, \ldots, K\}$ s.t. $\lim _{t \rightarrow f_{i}} C\left(P_{\bar{k}}, J, t\right)=\emptyset$, i.e., there exists a machine $\bar{k}$ which is free at any $t$ close enough to $f_{i}$. Thus, recalling our induction hypothesis (3), we may write:

$$
\sum_{\substack{j \in P^{k_{i}} \\ f_{j}<f_{i}}} y_{j}=\sum_{\substack{j \in P^{\bar{k}} \\ f_{j}<f_{i}}} y_{j}=\sum_{S_{g}: f_{i_{g}}<f_{i}} w_{g} .
$$

By contradiction, suppose now that $y_{i}>y_{i}^{w}$ (the case $y_{i}<y_{i}^{w}$ is similar). Then one can use (3)-(4) to write:

$$
\sum_{\substack{j \in P^{k_{i}} \\ f_{j} \leq f_{i}}} y_{j}=y_{i}+\sum_{\substack{j \in P^{k_{i}} \\ f_{j}<f_{i}}} y_{j}>\sum_{\substack{j \in P^{\bar{k}} \\ f_{j} \leq f_{i}}} y_{j} .
$$

Using Lemma 2 in (5) thus gives $\sum_{\substack{j \in P^{k_{i}} \\ f_{j}>f_{i}}} y_{j}<\sum_{\substack{j \in P^{\bar{k}} \\ f_{j}>f_{i}}} y_{j}$. Letting $P_{+}^{\bar{k}} \equiv\{j \in$ $\left.P^{\bar{k}}: f_{j}>f_{i}\right\} \neq \emptyset$ and $P_{-}^{k_{i}} \equiv\left\{j \in P^{k_{i}}: f_{j} \leq f_{i}\right\}$, and recalling from Lemma 2 that $\sum_{j \in P_{-}^{k_{i}}} y_{j}+\sum_{j \in P^{k_{i}} \backslash P_{-}^{k_{i}}} y_{j}=1=\sum_{j \in P_{+}^{\bar{k}}} y_{j}+\sum_{j \in P^{\bar{k}} \backslash P_{+}^{\bar{k}}} y_{j}$, one can then see that we have $\sum_{j \in P_{-}^{k_{i}} \cup P_{+}^{\bar{k}}} y_{j}=\sum_{j \in P_{-}^{k_{i}}} y_{j}+\sum_{j \in P_{+}^{\bar{k}}} y_{j}>1$. But this is a contradiction because $P_{-}^{k_{i}} \cup P_{+}^{\bar{k}}$ is a compatible coalition (for which core stability requires $\left.\sum_{j \in P_{-}^{k_{i}} \cup P_{+}^{\bar{k}}} y_{j} \leq 1\right)$. 
If we assume instead that $y_{i}<y_{i}^{w}$, the contradiction obtains in a similar way with the compatible set $P_{+}^{k_{i}} \cup P_{-}^{\bar{k}}$.

Case 2: Suppose that $f_{i}=f_{i_{g}}$ for some $g=1, \ldots, \bar{g}$. The desired result $\left(y_{i}=y_{i}^{w}\right)$ obtains by definition of $w_{g}$ if $i=i_{g}$. It hence follows from (3) that $\sum_{j \in P^{k_{i}: f_{j} \leq f_{i_{g}}}} y_{j}=w_{1}+\ldots+w_{g}$.

Assume now that $i \neq i_{g}$ and $f_{i}=f_{i_{g}}$. Then one can see that we have a new efficient allocation of the production in $\hat{P}=\left\{\hat{P}^{1}, \ldots, \hat{P}^{K}\right\}$ such that $\hat{P}^{k}=P^{k}$ if $k \notin\left\{k_{i}, k_{i_{g}}\right\} ; \hat{P}^{k_{i}}=\left(P^{k_{i}} \cup\left\{j \in P^{k_{i}}: f_{j} \leq f_{i_{g}}\right\}\right) \backslash\left\{j \in P^{k_{i}}: f_{j} \leq f_{i_{g}}\right\}$; and $\hat{P}^{k_{i_{g}}}=\left(P^{k_{i_{g}}} \cup\left\{j \in P^{k_{i}}: f_{j} \leq f_{i_{g}}\right\}\right) \backslash\left\{j \in P^{k_{i_{g}}}: f_{j} \leq f_{i_{g}}\right\}$. Applying Lemma 2 to $\hat{P}$ and $P$ thus gives: $y_{P^{k_{i}}}+\sum_{j \in P^{k_{i}: f_{j} \leq f_{i_{g}}}} y_{j}-\sum_{j \in P^{k_{i g}: f_{j} \leq f_{i_{g}}}} y_{j}=1=y_{P^{k_{i}}}$. It hence comes that $y_{i}+\sum_{j \in P^{k_{i}: f_{j} \leq s_{i}}} y_{j}=\sum_{j \in P^{k_{i}: f_{j} \leq f_{i}}} y_{j}=\sum_{j \in P^{k_{i}}: f_{j} \leq f_{i_{g}}} y_{j}=$ $w_{1}+\ldots+w_{g}$; and combining with (3), we get: $y_{i}=\sum_{g^{\prime}: i \in S_{g^{\prime}}} w_{g^{\prime}}=y_{i}^{w}$.

To conclude the proof, observe from Lemma 2 that our weight vector $w$ is well defined: $\sum_{g=1}^{\bar{g}} w_{g}=\sum_{j \in P^{1}} y_{j}=\ldots=\sum_{j \in P^{K}} y_{j}=1$. Moreover, we have $w_{g} \geq 0, \forall g=1, \ldots, \bar{g}$. Indeed, given that $y \in \operatorname{Core}(N, J)$, we have $y_{i_{1}}=w_{1} \geq 0$; and writing $w_{g}<0$ (for some $g=2, \ldots, \bar{g}$ ) leads to a contradiction, since the compatible coalition $S=\left\{i \in P_{k_{i_{g-1}}} \backslash\left\{i_{g}\right\}: f_{i} \leq f_{i_{g-1}}\right\} \cup\left\{i \in P_{k_{i_{g}}}: f_{i}>f_{i_{g}}\right\}$ then pays a joint share $y_{S}=\sum_{g^{\prime} \neq g} w_{g^{\prime}}=\sum_{g^{\prime}=1}^{\bar{g}} w_{g^{\prime}}-w_{g}=1-w_{g}>1=\bar{c}^{J}(S)$.

\subsection{Two distinguished cost sharing rules}

We now introduce two intuitive cost sharing rules that always pick allocations in the core $\mathcal{Y}(N, J)$, and that use the concept of proportonality. First, we define the peak-demand rule, $y^{P D}(N, J)$, by letting $w_{S}=\frac{1}{\bar{g}}$ for all $S \in G^{P D}$. We thus obtain a rule that shares costs in proportion to the number of peak-demand intervals an agent belongs to.

Definition 4 The peak-demand rule $y^{P D}$ is defined as $y_{i}^{P D}(N, J)=\frac{g_{i}^{P D}}{\bar{g}}$ for all $i \in N$ and corresponds to the allocation $y^{w}$ such that $w_{S}=\frac{1}{\bar{g}}$ for all $S \in G^{P D}$.

Next, we define a rule that takes into account the lengths of the respective peak-demand intervals. For all $S \in G^{P D}$, let $L(S)=\min _{i \in S} f_{i}-\max _{i \in S} s_{i}$ be the length of the peak-demand interval of coalition $S$. The peak-interval rule, $y^{P I}(N, J)$, is then obtained by letting $w_{S}=\frac{L(S)}{\sum_{T \in G^{P D}} L(T)}$.

Definition 5 The peak-interval rule $y^{P I}$ is defined as $y_{i}^{P I}(N, J)=\frac{\sum_{S \in G_{i}^{P D}} L(S)}{\sum_{T \in G^{P D}} L(T)}$ for all $i \in N$; and it corresponds to the allocation $y^{w}$ such that $w_{S}=\frac{L(S)}{\sum_{T \in G^{P D} L(T)}}$ for all $S \in G^{P D}$. 
Example 3 Recall the problem of Examples 1-2: $G^{P D}=\{\{2,4\},\{3,4\},\{3,5\}\}$ and it follows that $g^{P D}=(0,1,2,2,1)$. One thus gets $y^{P D}=\left(0, \frac{1}{3}, \frac{2}{3}, \frac{2}{3}, \frac{1}{3}\right)$ as the allocation recommended by the peak-demand rule.

We have $L(\{2,4\})=L(\{3,4\})=0.1$, while $L(\{3,5\})=0.2$. Thus, the peak-interval rule gives $y^{P I}=\left(0, \frac{1}{4}, \frac{3}{4}, \frac{1}{2}, \frac{1}{2}\right)$.

Notice that under the peak-demand rule, agents 2 and 5 pay the same share, as they both belong to one peak-demand coalition. However, in the peak-interval rule, agent 5 pays the same amount as agent 4 , as both have a job in a peakdemand interval for a length of 0.2. Agent 2, for which only 0.1 of her job is during a peak-interval, pays half of what agent 5 is paying.

\subsection{Characterizations}

In this subsection we propose axiomatic characterizations of the peak-demand rule and the peak-interval rule. We first formally define the property of Core Selection, which requires rules to always provide core allocations.

Core Selection: For all $(N, J), y(N, J) \in \operatorname{Core}(N, J)$.

Next, we define a property that applies to groups: if two distinct groups have the same number of agents and jobs of equal length, with no intersections with anybody else, then they play a similar role and their members should all pay the same cost share. The formal definition is as follows.

We say that two agents $i, j \in N$ are symmetric if $s_{i}=s_{j}$ and $f_{i}=f_{j}$. We say that two groups of agents $S, T \in 2^{N}$ are symmetric if

a) $|S|=|T|$

b) Any $i, j \in S$ are symmetric; and any $k, l \in T$ are symmetric.

c) $f_{i}-s_{i}=f_{j}-s_{j}$, for all $i \in S, j \in T$.

d) $\left[s_{i}, f_{i}\right) \cap\left[s_{k}, f_{k}\right)=\emptyset=\left[s_{j}, f_{j}\right) \cap\left[s_{k}, f_{k}\right)$, for all $i \in S, j \in T, k \in N \backslash(S \cup T)$.

In words, two agents are symmetric if they have the same starting and finishing times. Two groups are symmetric if a) they contain the same number of agents, b) each group is composed of symmetric agents, c) agents in both groups have jobs of the same length and d) each agent in either group is compatible with any agent not in the two groups.

It is a natural fairness requirement to impose that symmetric agents pay the same share, as well as members of symmetric groups.

Symmetry: In $(N, J)$, if $i, j \in N$ are symmetric agents, then $y_{i}(N, J)=$ $y_{j}(N, J)$.

Strong Symmetry: In $(N, J)$, if $S, T \subset N$ are symmetric groups, then $y_{i}(N, J)=y_{j}(N, J)$ for all $i, j \in S \cup T$.

Note that Symmetry is implied by Strong Symmetry. In addition, we draw the reader's attention to the fact that Strong Symmetry can also be interpreted as a fairness axiom about time neutrality. Indeed, the two groups $S, T$ in its definition are identical in all respects, except for the time at which their jobs are executed (e.g., morning vs afternoon). If Strong Symmetry is violated for 
two such groups, then it means that the considered cost sharing rule is not time neutral in the sense that jobs that have exactly the same characteristics (length, number of competing jobs, etc.) are charged different prices simply because they occur at different times of the day.

Next, we define two versions of No Merging No Splitting, which are designed to eliminate incentives for agents to strategically merge or split their jobs so as to lower their cost shares. The two versions differ because of the respective situations in which they apply. No Merging No Splitting states that the shares of other agents stay invariant only if the set of peak-demand coalitions is unchanged. This limits the domain of application of No Merging No Splitting. On the other hand, Strong No Merging No Splitting does not have this restriction.

We say that the problem $\left(N^{\prime}, J^{\prime}\right)$ is a splitting manipulation of the problem $(N, J)$ by agent $i \in N$ if: (a) $N^{\prime}=(N \backslash i) \cup\left\{i_{a}, i_{b}\right\}$, (b) $\left(s_{j}, f_{j}\right)=\left(s_{j}^{\prime}, f_{j}^{\prime}\right)$ for all $j \in N \backslash i,(\mathrm{c})\left(s_{i_{a}}^{\prime}, f_{i_{a}}^{\prime}\right) \cup\left(s_{i_{b}}^{\prime}, f_{i_{b}}^{\prime}\right)=\left(s_{i}, f_{i}\right)$ and $\left(s_{i_{a}}^{\prime}, f_{i_{a}}^{\prime}\right) \cap\left(s_{i_{b}}^{\prime}, f_{i_{b}}^{\prime}\right)=\emptyset$.

Equivalently, when the conditions (a)-(c) are satisfied, we also say that $(N, J)$ is a merging manipulation of the problem $\left(N^{\prime}, J^{\prime}\right)$ by the agents $i_{a}, i_{b}$.

No Merging No Splitting: Suppose that the problem $\left(N^{\prime}, J^{\prime}\right)$ is a splitting manipulation of the problem $(N, J)$ by agent $i \in N$; and $\left|G^{P D}\left(J^{\prime}\right)\right|=$ $\left|G^{P D}(J)\right|$. Then $y_{i}(N, J)=y_{i_{a}}\left(N^{\prime}, J^{\prime}\right)+y_{i_{b}}\left(N^{\prime}, J^{\prime}\right)$ and $y_{k}(N, J)=y_{k}\left(N^{\prime}, J^{\prime}\right)$ for all $k \in N \backslash i$.

Strong No Merging No Splitting: Suppose that the problem $\left(N^{\prime}, J^{\prime}\right)$ is a splitting manipulation of the problem $(N, J)$ by agent $i \in N$. Then $y_{i}(N, J)=$ $y_{i_{a}}\left(N^{\prime}, J^{\prime}\right)+y_{i_{b}}\left(N^{\prime}, J^{\prime}\right)$ and $y_{k}(N, J)=y_{k}\left(N^{\prime}, J^{\prime}\right)$ for all $k \in N \backslash i$.

It can be argued that changes to the set of peak-demand coalitions (under which Strong No Merging No Splitting applies, but not No Merging No Splitting) are easy to detect: any stable cost sharing rule uses peak-demand coalitions to assign cost shares. Therefore, it may be argued that we do not need to explicitly rule out such strategic manipulations (as they would be easier to detect).

Example 4 We revisit Example 1. Agent 2 has a job from 0.2 to 0.4 , and belongs to a peak-demand coalition with agent 4 between 0.3 and 0.4 . We consider two possible splitting manipulations.

Suppose first that agent 2 splits into agents $2 a$ and $2 b$, with respective jobs (0.2, 0.3) and $(0.3,0.4)$. The condition for NMNS is satisfied, as agent 4 has an intersection with $2 b$ but not with 2a. Therefore, following that split, both NMNS and $S N M N S$ prescribe that $y_{2 a}\left(N^{\prime}, J^{\prime}\right)+y_{2 b}\left(N^{\prime}, J^{\prime}\right)=y_{2}(N, J)$ and $y_{i}\left(N^{\prime}, J^{\prime}\right)=$ $y_{i}(N, J)$ otherwise. We can verify that we have $y^{P D}\left(N^{\prime}, J^{\prime}\right)=\left(0,0, \frac{1}{3}, \frac{2}{3}, \frac{2}{3}, \frac{1}{3}\right)$ and $y^{P I}\left(N^{\prime} J^{\prime}\right)=\left(0,0, \frac{1}{4}, \frac{3}{4}, \frac{1}{2}, \frac{1}{2}\right)$ so that this manipulation is thwarted by both rules.

Suppose next that agent 2 splits into agents $2 c$ and $2 d$ with respective job (0.2, 0.35) and $(0.35,0.4)$. The condition for NMNS is not satisfied, as agent 4 now has an intersection that creates a peak-demand coalition with both agents $2 c$ and 2d. Therefore, following that split, NMNS puts no constraints on the shares but $S N M N S$ still prescribes that $y_{2 c}\left(N^{\prime}, J^{\prime}\right)+y_{2 d}\left(N^{\prime}, J^{\prime}\right)=y_{2}(N, J)$ and 
$y_{i}\left(N, J^{\prime}\right)=y_{i}(N, J)$ otherwise. We can verify that we have $y^{P D}\left(N^{\prime}, J^{\prime}\right)=$ $\left(0, \frac{1}{4}, \frac{1}{4}, \frac{1}{2}, \frac{3}{4}, \frac{1}{4}\right)$ and $y^{P I}\left(N^{\prime} J^{\prime}\right)=\left(0, \frac{1}{8}, \frac{1}{8}, \frac{3}{4}, \frac{1}{2}, \frac{1}{2}\right)$ so that $y^{P D}$ fails SNMNS but $y^{P I}$ satisfies it.

In terms of merging, notice that neither NMNS nor SNMNS protects against the (non-credible threat of) merger of agent 2 with either agent 4 (because their jobs intersect) or agent 5 (because their merger would create the need for a third machine between 0.4 and 0.5). NMNS protects against a merger with agent 1 while SNMNS also protects against a merger with agent 3.

Finally, we introduce a property saying that if an agent changes the duration of her job without impacting any of the stand-alone costs, then it should not have any impact on the cost shares either.

Independence of Irrelevant Intervals: For all $(N, J)$ let $J^{\prime}=\left\{\left\{s_{k}, f_{k}\right\}_{k \in N \backslash\{i\}}\right.$, $\left.\left\{s_{i}-a, f_{i}+b\right\}\right\}$, with $a, b \geq 0$. Then, if $C^{J}=C^{J^{\prime}}, y(N, J)=y\left(N, J^{\prime}\right)$.

In other words, the cost shares should depend only on the cooperative cost game generated by the job scheduling problem. Combining these axioms, one obtains the following characterization result.

Theorem 2 A rule y satisfies No Merging No Splitting, Independence of Irrelevant Intervals and Strong Symmetry if and only if it is the peak-demand rule.

Proof. It is easy to verify that the peak-demand rule satisfies No Merging No Splitting, Independence of Irrelevant Intervals and Strong Symmetry. We show that it is the only rule satisfying the properties.

Suppose that $y$ satisfies No Merging No Splitting, Independence of Irrelevant Intervals and Strong Symmetry.

Step 1: Use of No Merging No Splitting. For each $i \in N$, let $A^{i}=$ $\left\{S^{i, 1}, \ldots, S^{i, K_{i}}\right\}$, with each $S^{i, k} \subseteq N \backslash i$, be such that

- $t_{S^{i, k}}^{\min }<t_{S^{i, l}}^{\min }$ if $k<l$;

- $t_{S^{i, k}}^{\max }=t_{S^{i, k+1}}^{\min }$ for all $k=1, \ldots, K_{i}-1$;

- $t_{S^{i, 1}}^{\min }=s_{i}$;

- $t_{S^{i}, K_{i}}^{\max }=f_{i}$;

- $S^{i, k} \neq S^{i, k+1}$ for all $k=1, \ldots, K_{i}-1$;

- $C(N, J, t)=S^{i, k} \cup i$ for all $t \in\left[t_{S^{i}, k}^{\min }, t_{S^{i, k}}^{\max }[\right.$.

In words, $A^{i}$ gives the list of coalitions $S^{i, k}$ that are incompatible with $i$ at some given time $t$, since $S^{i, k}$ changes as $t$ varies from $s_{i}$ to $f_{i}$.

We create new agents in the following manner: for all $S^{i, k} \in A^{i}$, create agent $i_{k}$ such that $s_{i_{k}}=t_{S^{k}}^{\min }$ and $f_{i_{k}}=t_{S^{k}}^{\max }$. In words, we are creating multiple agents, one for each coalition $S^{i, k}$ that is incompatible with $i$. 
Let $N^{i}=\left\{i_{k}\right\}_{k=1}^{K_{i}}$ be the set of agents created for agent $i$. Then $N^{\prime}=$ $N^{1} \cup \ldots \cup N^{n}$ contains all agents created via this procedure; and the list of their jobs is $J^{\prime}=\left\{\left(s_{i_{k}}, f_{i_{k}}\right)\right\} \begin{aligned} & i=1, \ldots, n \\ & k=1, \ldots, K_{i}\end{aligned}$.

By (multiple applications of) No Merging No Splitting, it follows that, for all $i \in N$,

$$
y_{i}(N, J)=\sum_{k=1}^{K_{i}} y_{i_{k}}\left(N^{\prime}, J^{\prime}\right) .
$$

Step 2: Use of Independence of Irrelevant Intervals. For all $l=$ $1, \ldots, \bar{g}$, let

$$
z_{l}=\min _{j \in N} \min _{\substack{k=1, \ldots, K_{j} \\\left|S^{j, k}\right|=l-1}} t_{S^{j, k}}^{\max }-t_{S^{j, k}}^{\min }
$$

be the shortest length of an interval for an incompatible coalition of size $l$. For all $i \in N$ and $k=1, \ldots, K_{i}$, let $f_{i_{k}}^{\prime}=s_{i_{k}}+z_{\left|S^{i, k}\right|+1}$, with other start times remaining unchanged. Formally, for all $i \in N$, let $J^{\prime \prime i}=\left\{\left(s_{i_{k}}, s_{i_{k}}+z_{\left|S^{i, k}\right|+1}\right)\right\}_{k=1}^{K_{i}}$; and define $J^{\prime \prime}=\left\{J^{\prime \prime 1}, \ldots, J^{\prime \prime n}\right\}$. By Independence of Irrelevant Intervals, $y\left(N^{\prime}, J^{\prime}\right)=$ $y\left(N^{\prime}, J^{\prime \prime}\right)$ and thus for all $i \in N$,

$$
y_{i}(N, J)=\sum_{k=1}^{K_{i}} y_{i_{k}}\left(N^{\prime}, J^{\prime \prime}\right) .
$$

Step 3: Use of Strong Symmetry. Let $G^{k}$ be the set of coalitions $S \subseteq N^{\prime}$ such that $\bar{c}^{J^{\prime \prime}}(S)=|S|=k$, for all $k=1, \ldots, \bar{g}$. We verify that in the problem $\left(N^{\prime}, J^{\prime \prime}\right)$, the four conditions of Strong Symmetry are satisfied for any pair of distinct coalitions $S, T \in G^{k}$. one coalition $S \in G^{k}$, so $S \cap T=\emptyset$.

First, since $S, T \in G^{k}$, we have $|S|=|T|$, and condition a) is satisfied.

Second, in $J^{\prime \prime}$, we have set in Step $1 s_{i}=t_{S}^{\min }$ and in Step $2 f_{i}=t_{S}^{\min }+z_{k}$, for all $i \in S$. Thus, $S$ is composed of symmetric agents. In the same manner we have set in Step $1 s_{i}=t_{T}^{\min }$ and in Step $2 f_{i}=t_{T}^{\min }+z_{k}$, for all $i \in T$. Thus, $S$ is also composed of symmetric agents, and condition b) is satisfied. It follows that if $i \in S$ and $j \in T$, we have $f_{i}-s_{i}=z_{k}=f_{j}-s_{j}$, and condition c) is satisfied,

Finally, since all members of $S$ have the same interval and $S \in G^{k}$, any intersection with any other job would contradict this. The same reasoning applies for $T$. Thus condition d) is satisfied.

Thus, $S$ and $T$ are symmetric groups. We thus have that $y_{i}=y_{j}$ for all $i, j \in$ $S \cup T$. In our case, it means that for all $k=1, \ldots, K_{i}, y_{i_{k}}\left(N^{\prime}, J^{\prime \prime}\right) \equiv \alpha_{\left|S^{i, k}\right|+1}$.

Step 4: Zero share for non-peak demands. We next show that $\alpha_{l}=0$ for all $l=1, \ldots, \bar{g}-1$.

Fix $l \in\{1, \ldots, \bar{g}-1\}$ and for all $S \in G^{l}$, and all $i \in S$, split the agent in two, $i_{a}$ and $i_{b}$, dividing the job in two two equal parts: let $\hat{s}_{i_{a}}=s_{i}^{\prime}, \hat{f}_{i_{a}}=\hat{s}_{i_{b}}=\frac{s_{i}^{\prime}+f_{i}^{\prime \prime}}{2}$ and $\hat{f}_{i_{b}}=f_{i}^{\prime \prime}$ for all $i \in S$. Let $\hat{N}$ and $\hat{J}$ be the corresponding new sets of agents and jobs. By No Merging No Splitting, we have $y_{i_{a}}(\hat{N}, \hat{J})+y_{i_{b}}(\hat{N}, \hat{J})=\alpha_{l}$. By 
Strong Symmetry, $y_{i_{a}}(\hat{N}, \hat{J})=y_{i_{b}}(\hat{N}, \hat{J})=\frac{\alpha_{l}}{2}$. For all $i \in S$, split the agent $i_{b}$ into two further agents, $i_{b 1}$ and $i_{b 2}$, dividing the job in two equal parts: let $\check{s}_{i_{b 1}}=\hat{s}_{i_{b}}, \check{f}_{i_{b 1}}=\check{s}_{i_{b 2}}=\frac{\hat{s}_{i_{b}}+\hat{f}_{i_{b}}}{2}$ and $\check{f}_{i_{b 2}}=\hat{f}_{i_{b}}$ for all $i \in S$. Let $\check{N}$ and $\check{J}$ be the corresponding new sets of agents and jobs. By the same argument as above, we obtain $y_{i_{b 1}}(\check{N}, \check{J})=y_{i_{b 2}}(\check{N}, \check{J})=\frac{\alpha_{l}}{4}$.

Next, for all $i \in S$, remove the second half of the job of agent $i_{a}: \tilde{s}_{i_{a}}=\hat{s}_{i_{a}}$ and $\tilde{f}_{i_{a}}=\frac{\hat{s}_{i_{a}}+\hat{f}_{i_{a}}}{2}$. Let $\tilde{J}$ be the corresponding new set of jobs. By Independence of Irrelevant Intervals, $y_{i}(\check{N}, \tilde{J})=y_{i}(\check{N}, \breve{J})$ for all $i \in \check{N}$. By Strong Symmetry, $\frac{\alpha_{l}}{2}=y_{i_{a}}(\check{N}, \check{J})=y_{i_{b 1}}(\check{N}, \check{J})=\frac{\alpha_{l}}{4}$, which implies that $\alpha_{l}=0$ for all $l=$ $1, \ldots, \bar{g}-1$. Thus,

$$
y_{i}(N, J)=\sum_{\substack{k \in\left\{1, \ldots, K_{i}\right\} \\\left|S^{i, k}\right|=\bar{g}-1}} y_{i_{k}}\left(N^{\prime}, J^{\prime \prime}\right)=\sum_{\substack{k \in\left\{1, \ldots, K_{i}\right\} \\\left|S^{i, k}\right|=\bar{g}-1}} \alpha_{\bar{g}} .
$$

Step 5: Use of budget balance. The total cost is $\bar{u}$. We have $\bar{g}$ peakdemand coalitions containing exactly $\bar{u}$ agents each, thus $\alpha_{\bar{g}}=\frac{1}{\bar{g} \bar{u}} \bar{u}=\frac{1}{\bar{g}}$ for all $i \in N$ and all $k$. We thus obtain that for all $i \in N$,

$$
y_{i}(N, J)=\sum_{\substack{k \in\left\{1, \ldots, K_{i}\right\} \\\left|S^{i, k}\right|=\bar{g}-1}} \frac{1}{\bar{g}}=\frac{g_{i}^{P D}}{\bar{g}}=y_{i}^{P D}(N, J) .
$$

We obtain an axiomatization of the peak-interval rule by replacing Independence of Irrelevant Intervals by Core Selection, and strengthening No Splitting No Merging into Strong No Merging No Splitting.

Theorem 3 A rule y satisfies Core Selection, Strong No Merging No Splitting and Strong Symmetry if and only if it is the peak-interval rule.

Proof. It is easy to verify that the peak-interval rule satisfies Core Selection, Strong No Merging No Splitting and Strong Symmetry. We show that it is the only rule satisfying the properties.

Suppose that a rule $y$ meets Core Selection, Strong No Merging No Splitting and Strong Symmetry; and fix a problem $(N, J)$. Our first step consists in splitting each peak-demand intervals in multiple intervals of size $q$, with $q$ being the largest number such that for all $S \in G^{P D}(J)$, there exists $k^{S} \in \mathbb{N}$ satisfying $L(S)=k^{S} q$. One can then create multiple agents, $k^{S}$ for each peak-demand coalition $S$ that $i$ belongs to, with each agent having a job of length $q$. We also create one agent for each interval on which $i$ is demanding, but not part of a peak-demand coalition.

Step 1: Use of Strong No Merging No Splitting. For each $i \in N$, label coalitions in $G_{i}^{P D}$ such that $G_{i}^{P D}=\left\{S^{1}, \ldots, S^{g_{i}^{P D}}\right\}$ and $t_{S^{k}}^{\min }<t_{S^{l}}^{\min }$ if $k<l$. We create new agents in the following manner: for all $S^{l} \in G_{i}^{P D}$, create $k^{S^{l}}$ agents $i_{l_{k}}$ and one agent $i_{\bar{l}}$ such that $s_{i_{l_{1}}}=t_{S^{l}}^{\text {min }}, s_{i_{l_{k}}}=s_{i_{l_{k-1}}}+q$ for 
$k=2, \ldots, k^{S^{l}}, f_{i_{l_{k}}}=s_{i_{l_{k}}}+q$ for $k=1, \ldots, k^{S^{l}}, s_{i_{\bar{l}}}=t_{S^{l-1}}^{\max }$ and $f_{i_{\bar{l}}}=t_{S^{l}}^{\min }$, with $t_{S^{0}}^{\max }=s_{i}$. Create an additional agent $i \frac{}{g_{i}^{P D}+1}$ such that $s_{i \frac{g_{i}^{P D}+1}{}}=t_{S^{g_{i}^{P D}}}^{\max }$ and $f_{i \frac{g_{i}^{P D}+1}{}}=f_{i}$. Notice that for some $k$, we might have that $f_{i_{\bar{k}}}=s_{i_{\bar{k}}}$, in which case we can say that the agent $i_{\bar{k}}$ is not created.

Let $N^{i_{l}}=\left\{i_{l_{k}}\right\}_{k=1}^{k^{S^{l}}}$ be the set of agents created for agent $i$ to cover her demand with peak-demand coalition $S^{l}$. Let $J^{i_{l}}=\left\{\left(s_{i_{l_{k}}}, f_{i_{l_{k}}}\right)\right\}_{k=1}^{k^{S^{l}}}$ be the set of jobs of the agents in $N^{i_{l}}$.

Let $N^{i}=\left\{\left\{N^{i_{l}}, i_{\bar{l}}\right\}_{l=1}^{g_{i}^{P D}}, i \frac{}{g_{i}^{P D}+1}\right\}$ be the set of agents created for agent $i$. Let $J^{i}=\left\{\left\{J^{i_{l}}\right\}_{l=1}^{g_{i}^{P D}},\left\{\left(s_{i_{\bar{l}}}, f_{i_{\bar{l}}}\right)\right\}_{l=1}^{g_{i}^{P D}+1}\right\}$ be the set of jobs of the agents in $N^{i}$.

Let $N^{\prime}=\left\{N^{1} \cup \ldots \cup N^{n}\right\}$ be the set of agents created for all agents and let $J^{\prime}=\left\{J^{1}, \ldots, J^{n}\right\}$ be the set of jobs for these agents.

By (multiple applications of) Strong No Merging No Splitting, we have that for all $i \in N$,

$$
y_{i}(N, J)=\sum_{l=1}^{g_{i}^{P D}} \sum_{k=1}^{k^{S^{l}}} y_{i_{l_{k}}}\left(N^{\prime}, J^{\prime}\right)+\sum_{l=1}^{g_{i}^{P D}+1} y_{i_{\bar{l}}}\left(N^{\prime}, J^{\prime}\right) .
$$

Step 2: Use of Core Selection. By Theorem 1, $y_{i_{\bar{l}}}=0$ in any core allocation for all $i \in N$ and all $l$. We thus have that for all $i \in N$,

$$
y_{i}(N, J)=\sum_{l=1}^{g_{i}^{P D}} \sum_{k=1}^{k^{S^{l}}} y_{i_{k}}\left(N^{\prime}, J^{\prime}\right)
$$

\section{Step 3: Use of Strong Symmetry.}

Let us check, that in the problem $\left(N^{\prime}, J^{\prime}\right)$, the four conditions of Strong Symmetry are satisfied for any pair of distinct coalitions $S, T \in G^{P D}\left(J^{\prime}\right)$.

First, since $S, T \in G^{P D}\left(J^{\prime}\right)$, we have $|S|=|T|$, and condition a) is satisfied.

Second, for $S \in G^{P D}\left(J^{\prime}\right)$ let $\bar{S} \subset N$ be such that $\left(s_{i}, f_{i}\right) \cap\left(s_{j}, f_{j}\right) \neq \emptyset$ for all $i \in S$ and $j \in \bar{S}$ (i.e., $S$ is one of the pieces we have divided $\bar{S}$ into in Step 1). Then, from Step 1 , we have that if $i, j \in S, s_{i}=s_{j}=t_{\bar{S}}^{\min }+(k-1) q$ and $f_{i}=f_{j}=t_{\bar{S}}^{m i n}+k q$, for some $k \in 1, \ldots, k^{S}$. Thus all agents in $S$ have the same job and are thus symmetric agents.

The same logic as above applies for $T \in G^{P D}\left(J^{\prime}\right)$. Thus all agents in $T$ are symmetric agents, and condition b) is satisfied. Since agents in both groups have a job of length $q$, condition c) is satisfied.

Finally, since $S$ is a peak-demand coalition, any intersection with any other job would increase the size of the peak-demand coalition, a contradiction. The same reasoning applies for $T$. Thus condition d) is satisfied.

Thus, $S$ and $T$ are symmetric groups and $y_{i_{l_{k}}}\left(N^{\prime}, J^{\prime}\right) \equiv \alpha$ for all $i \in N$, $l=1, \ldots, g_{i}^{P D}$ and $k=1, \ldots, k^{S^{l}}$. 
Step 4: Use of budget balance. Total cost is $\bar{u}$. In $J^{\prime}$, we have $\sum_{T \in G^{P D}(J)} k^{T}=\sum_{T \in G^{P D}(J)} \frac{L(T)}{q}$ peak-demand coalitions, each containing exactly $\bar{u}$ agents each, thus $y_{i_{l_{k}}}=\frac{1}{\bar{u} \sum_{T \in G^{P D}(J)} \frac{L(T)}{q}} \bar{u}=\frac{1}{\sum_{T \in G^{P D}(J)} \frac{L(T)}{q}}$ for all $i \in N$ and all $k, l$. We thus obtain that for all $i \in N$,

$$
\begin{aligned}
y_{i}(N, J) & =\sum_{l=1}^{g_{i}^{P D}} \sum_{k=1}^{k^{S^{l}}} \frac{1}{\sum_{T \in G^{P D}(J)} \frac{L(T)}{q}} \\
& =\sum_{l=1}^{g_{i}^{P D}} \frac{k^{S^{l}}}{\sum_{T \in G^{P D}(J)} \frac{L(T)}{q}}=\sum_{l=1}^{g_{i}^{P D}} \frac{\frac{L\left(S^{l}\right)}{q}}{\sum_{T \in G^{P D}(J)} \frac{L(T)}{q}} \\
& =\frac{\sum_{S \in G_{i}^{P D}} \frac{L(S)}{q}}{\sum_{T \in G^{P D}(J)} \frac{L(T)}{q}}=\frac{\sum_{S \in G_{i}^{P D}} L(S)}{\sum_{T \in G^{P D}(J)} L(T)}=y_{i}^{P I}(N, J) .
\end{aligned}
$$

\section{Unanimity Lower Bound: the Shapley value}

One striking feature of core allocations for the job scheduling problem is that any agent that does not belong to a peak-demand coalition is not assigned any cost. But, since every agent (or coalition) would pay at least the full cost of one machine if he stood alone, it seems very natural to assume that everybody is equally responsible for the cost of the first machine used. Thus, regardless of compatibility issues, it is natural and fair to require that all agents should pay at least $\frac{1}{n}$, an equal share of the cost of the first machine.

This concept has been used in various environments - see Moulin (1990), Maniquet (1996) and Hougaard and Moulin (2014); and we follow the literature in calling the property Unanimity Lower Bound.

Unanimity Lower Bound: For all $(N, J)$ and $i \in N, y_{i}(N, J) \geq \frac{1}{n}$.

Given Theorem 1, the following result is immediate:

Corollary 1 There exists no rule satisfying both Core Selection and Unanimity Lower Bound.

\subsection{The Shapley value}

If one is willing to sacrifice Core Selection in order to use a rule satisfying Unanimity Lower Bound, a natural candidate is the Shapley value, formally defined as follows. For all $i \in N$,

$$
y_{i}^{S h}(N, J)=\sum_{S \in N \backslash i} \frac{|S| !(n-|S|-1) !}{n !}\left(\bar{c}^{J}(S \cup i)-\bar{c}^{J}(S)\right) .
$$


In the context of the job scheduling problem, we can simplify this general formula. In particular, $\left(\bar{c}^{J}(S \cup i)-\bar{c}^{J}(S)\right)$ can only take two values: zero or one. Define $W I_{i}(J)=\left\{S \in N \backslash i \mid \bar{c}^{J}(S \cup i)=\bar{c}^{J}(S)+1\right\}$ as the set of coalitions that are weakly-incompatible with $i$, i.e. such that adding $i$ to these coalitions requires an extra machine. Note that if $S \in W I_{i}(J), i$ might still be compatible with some agents in $S$. For instance, if $S=\{j, k\}$, if $i$ is compatible with $j$ and incompatible with $k$, and $j, k$ are compatible with each other, then $i$ is weakly incompatible with $S$.

We obtain the following simplification of the expression of the Shapley value. For all $i \in N$,

$$
y_{i}^{S h}(N, J)=\sum_{S \in W I_{i}(J)} \frac{|S| !(n-|S|-1) !}{n !} .
$$

Example 5 In our running example, we have: $W I_{1}=\{\emptyset\}$,

$W I_{2}=\{\emptyset,\{4\},\{1,4\},\{4,5\},\{1,4,5\}\}$,

$W I_{3}=\{\emptyset,\{4\},\{5\},\{1,4\},\{1,5\},\{2,5\}\{4,5\},\{1,2,5\},\{1,4,5\}\}$,

$W I_{4}=\{\emptyset,\{2\},\{3\},\{1,2\},\{1,3\},\{2,3\}\{2,5\},\{1,2,3\},\{1,2,5\}\}$ and

$W I_{5}=\{\emptyset,\{3\},\{1,3\},\{2,3\},\{1,2,3\}\}$.

We thus obtain $y^{S h}=\left(\frac{1}{5}, \frac{11}{30}, \frac{8}{15}, \frac{8}{15}, \frac{11}{30}\right)$.

\subsection{Characterization}

We characterize the Shapley value by using the property of Equal Treatment, which says that if we add an incompatibility between two agents, they should see their cost shares affected in the same manner. The property is inspired by Myerson (1977) and Kar (2002), who use it in slightly different contexts. We need the following notation.

Denote $I(J)=\left\{(k, l) \in N \times N \mid\left(s_{k}, f_{k}\right) \cap\left(s_{l}, f_{l}\right) \neq \emptyset\right\}$. For $(k, l) \notin I(J)$, let $J^{+k l}$ be such that $I\left(J^{+k l}\right)=I(J) \cup(k, l)$.

Equal Treatment: For $(k, l) \in I(J), y_{k}(N, J)-y_{k}\left(N, J^{+k l}\right)=y_{l}(N, J)-$ $y_{l}\left(N, J^{+k l}\right)$

The property is combined with a very simple property that we call Full Incompatibility: if an agent is incompatible with all others, then he will require a machine just to execute her job, and he should pay the full cost of it.

Let $N^{F}(J)=\{i \in N \mid(i, j) \in I(J)$ for all $j \in N \backslash i\}$ be the set of agents who are incompatible with all other agents.

Full Incompatibility: If $k \in N^{F}(J)$, then $y_{k}(N, J)=1$.

Theorem 4 A rule satisfies Equal Treatment and Full Incompatibility if and only if it is the Shapley value.

Proof. It is easy to verify that the Shapley value satisfies Equal Treatment and Full Incompatibility. We show that it is the only rule satisfying these properties. 
We proceed by induction on the cardinality of $I(J)$.

Suppose that $|I(J)|=\frac{n(n-1)}{2}$. Then, $N^{F}(J)=N$ and $y_{i}(J)=1$ for all $i \in N$ by Full Incompatibility.

Suppose that we have shown that there is a unique rule satisfying the properties if $|I(J)| \geq m+1$. We show that it implies that there is a unique rule satisfying the properties if $|I(J)|=m$.

We proceed by contradiction, supposing that $y$ and $x$ both satisfy the properties and that $x(J) \neq y(J)$ when $|I(J)|=m$.

Take $(k, l) \notin I(J)$. Now

$$
\begin{aligned}
y_{k}(J)-y_{l}(J) & =y_{k}\left(J^{+k l}\right)-y_{l}\left(J^{+k l}\right) \text { (by Equal Treatment) } \\
& =x_{k}\left(J^{+k l}\right)-x_{l}\left(J^{+k l}\right) \text { (by induction hypothesis) } \\
& =x_{k}(J)-x_{l}(J) \text { (by Equal Treatment) }
\end{aligned}
$$

and thus $y_{k}(J)-x_{k}(J)=y_{l}(J)-x_{l}(J)$.

Take any other $(i, j) \notin I(J)$. Then, repeating the argument above, we obtain $y_{i}(J)-x_{i}(J)=y_{j}(J)-x_{j}(J)$.

For all $i \in N^{F}(J)$, we have that $y_{i}(J)=x_{i}(J)=1$ and thus that $y_{i}(J)-$ $x_{i}(J)=0$.

Claim: For all $i, j \in N \backslash N^{F}(J)$, there exists a sequence $i_{1}, \ldots, i_{K}$ with $i_{1}=i$ and $i_{K}=j$ such that $\left(i_{k}, i_{k+1}\right) \notin I(J)$ for all $k=1, \ldots, K-1$.

Suppose otherwise. Let $N^{*}=N \backslash N^{F}(J)$. Then, we must have $S$, with $i \in S$ and $j \in N^{*} \backslash S$, such that for all $j \in S$ and $k \in N^{*} \backslash S,(j, k) \in I(J)$. Since $i, j \notin N^{F}(J)$, there exists $l \in S$ and $m \in N^{*} \backslash S$ such that $(i, l),(j, m) \notin I(J)$. This imposes that $2 \leq|S| \leq\left|N^{*}\right|-2$.

Suppose without loss of generality that $s_{i}<s_{l}$. Since $i$ and $l$ are compatible, $f_{i} \leq s_{l}$. By definition of $S, j$ and $m$ are incompatible with both $i$ and $l$. Thus, $s_{j}, s_{m}<f_{i}$ and $f_{j}, f_{m}>s_{l}$. In other words, $\left[f_{i}, s_{l}\right] \in\left(s_{j}, f_{j}\right) \cap\left(s_{m}, f_{m}\right)$ and $j$ and $m$ are incompatible, contradicting our initial assumption. Thus, our claim is verified.

Given that for all $i, j \in N \backslash N^{F}(J)$, there exists a sequence $i_{1}, \ldots, i_{K}$ with $i_{1}=i$ and $i_{K}=j$ such that $\left(i_{k}, i_{k+1}\right) \notin I(J)$ for all $k=1, \ldots, K-1$, it is immediate that we obtain, by multiple applications of the argument above, that $y_{i}(J)-x_{i}(J)=y_{j}(J)-x_{j}(J)$.

We must have

$$
\begin{aligned}
\sum_{i \in N}\left[y_{i}(J)-x_{i}(J)\right] & =0 \text { (by budget balance) } \\
\sum_{i \in N^{*}}\left[y_{i}(J)-x_{i}(J)\right] & =0\left(\text { since } y_{i}(J)=x_{i}(J) \text { for } i \in N^{F}(J)\right) \\
y_{i}(J)-x_{i}(J) & =0 \forall i \in N^{*}, \text { since } y_{i}(J)-x_{i}(J)=y_{j}(J)-x_{j}(J) \forall i, j \in N^{*}
\end{aligned}
$$

Thus, $y(J)=x(J)$. 


\section{A compromise between Core Selection and Unanimity Lower Bound}

As seen in the previous section, Core Selection and Unanimity Lower Bound are incompatible. We propose in this section a compromise based on a weakening of the stability concept behind Core Selection.

Given that all coalitions would need to pay for at least one machine if they were to undertake the project by themselves, one can imagine that constraints are put on departing coalitions, with coalition $S$ being asked to pay $\frac{|S|}{n}$, its fair share of the "fixed" cost of 1 . We thus consider a modified version of stability that will be compatible with Unanimity Lower Bound. To this end, we need to generalize our job scheduling problems, now identified as $(N, J, a)$, where (just as before) $N$ and $J$ are respectively the set of agents and their profile of jobs. The new argument in the tuple $(N, J, a)$ is $a$, the constant cost of adding a new machine machine. For all $S \subseteq N$, let $C(S, J, a)=a \bar{c}^{J}(S)$ and $\tilde{C}(S, J, a)=\frac{|S|}{n}+C(S, J, a)$. The coalitional game $C(\cdot, J, a)$ thus comes from the problem with unit cost $a$ for machines, while $\tilde{C}(\cdot, J, a)$ adds to each coalition their fair share of the "fixed" cost of the first machine (which is 1).

We consider the game $\tilde{C}\left(S, J, \frac{\bar{c}^{J}(N)-1}{\bar{c}^{J}(N)}\right)$, where coalitions pay their fair share of the fixed cost of 1 but variable costs are rescaled so as to satisfy budget balance in the original problem $(N, J, 1)$.

Lemma $3 \tilde{C}\left(N, J, \frac{\bar{c}^{J}(N)-1}{\bar{c}^{J}(N)}\right)=C(N, J, 1)$.

Proof. We have that

$$
\begin{aligned}
\tilde{C}\left(N, J, \frac{\bar{c}^{J}(N)-1}{\bar{c}^{J}(N)}\right) & =\frac{n}{n}+\frac{\bar{c}^{J}(N)-1}{\bar{c}^{J}(N)} \bar{c}^{J}(N) \\
& =1-\frac{\bar{c}^{J}(N)}{\bar{c}^{J}(N)}+\bar{c}^{J}(N) \\
& =\bar{c}^{J}(N) .
\end{aligned}
$$

By extension, $\operatorname{Core}(N, J, a)=\left\{y \in \mathbb{R}_{+}^{N} \mid y_{N}=a \bar{c}^{J}(N)\right.$ and $\left.y_{S} \leq a \bar{c}^{J}(S)\right\}$. Let $\widetilde{\operatorname{Core}}(N, J)=\left\{y \in \mathbb{R}_{+}^{N} \mid y_{N}=\bar{c}^{J}(N)\right.$ and $\left.y_{S} \leq \tilde{C}\left(S, J, \frac{\bar{c}^{J}(N)-1}{\bar{c}^{J}(N)}\right)\right\}$. It is obvious that $y \in \operatorname{Core}(N, J, 1)$ if and only if ay $\in \operatorname{Core}(N, J, a)$, for all $a>0$. Thus, Theorem 1 allows to find all allocations in $\operatorname{Core}(N, J, a)$.

We formally define the Weak Stability property described above: we suppose that departing coalition $S$ would have to pay $\frac{|S|}{n}$ when it departs.

Weak Stability: For all problems $(N, J), y(N, J) \in \widetilde{\operatorname{Core}}(N, J)$.

While in our example the Shapley value is in $\widetilde{\operatorname{Core}}(N, J)$, this is not true in general. Consider a 3 player example in which agents 1 and 2 are compatible with each other, and agent 3 is incompatible with both. Thus $\bar{c}^{J}(N)=2$ and 
the Shapley value assigns shares $\left(\frac{1}{2}, \frac{1}{2}, 1\right)$. We can verify that $\tilde{C}\left(\{3\}, J, \frac{1}{2}\right)=$ $\frac{1}{2}+\frac{1}{3}<1=y_{3}^{S h}$.

However, there are rules satisfying Unanimity Lower Bound and Weak Stability. We obtain a large set of such rules by modifying the rules satisfying Core Selection, like our peak-demand and peak-interval rules.

For any rule $y$, we define the modification $\dot{y}$ by: $\dot{y}(N, J)=\frac{1}{n}+y\left(N, J, \frac{\bar{c}^{J}(N)-1}{\bar{c}^{J}(N)}\right)$, for all problems $(N, J)$ with unit cost of 1 .

Theorem 5 If $y(N, J)$ satisfies Core Selection, then $\dot{y}(N, J)$ satisfies Weak Stability and Unanimity Lower Bound.

Proof. Fix $(N, J$ and suppose that $y(N, J)$ satisfies Core Selection

Weak Stability: Fix $S \subseteq N$. We have that

$$
\begin{aligned}
\dot{y}_{S}(N, J) & =\frac{|S|}{n}+y_{S}\left(N, J, \frac{\bar{c}^{J}(N)-1}{\bar{c}^{J}(N)}\right) \\
& \left.\leq \frac{|S|}{n}+\frac{\bar{c}^{J}(N)-1}{\bar{c}^{J}(N)} \bar{c}^{J}(S) \text { (since } y \text { satisfies Core Selection) }\right) \\
& =\tilde{C}\left(S, J, \frac{\bar{c}^{J}(N)-1}{\bar{c}^{J}(N)}\right) .
\end{aligned}
$$

Unanimity Lower Bound: Since $y$ satisfies Core Selection, it follows that $y_{i}\left(N, J, \frac{\bar{c}^{J}(N)-1}{\bar{c}^{J}(N)}\right) \geq 0$ for all $i \in N$, in all core allocations. That is to say, $\dot{y}_{i}(N, J)=\frac{1}{n}+y_{i}\left(N, J, \frac{\bar{c}^{J}(N)-1}{\bar{c}^{J}(N)} \geq \frac{1}{n}\right.$, for all $i \in N$.

Since we know how to find rules satisfying Core Selection, we obtain a large family satisfying the two properties.

Example 6 We revisit our example. Since $\bar{c}^{J}(N)=2$, we consider a game in which the variable cost is $\frac{1}{2}$. We thus obtain $\dot{y}^{P D}=\left(\frac{1}{5}, \frac{1}{5}, \frac{1}{5}, \frac{1}{5}, \frac{1}{5}\right)+\left(0, \frac{1}{6}, \frac{1}{3}, \frac{1}{3}, \frac{1}{6}\right)=$ $\left(\frac{1}{5}, \frac{11}{30}, \frac{8}{15}, \frac{8}{15}, \frac{11}{30}\right)$ and $\dot{y}^{P I}=\left(\frac{1}{5}, \frac{1}{5}, \frac{1}{5}, \frac{1}{5}, \frac{1}{5}\right)+\left(0, \frac{1}{8}, \frac{3}{8}, \frac{1}{4}, \frac{1}{4}\right)=\left(\frac{1}{5}, \frac{13}{40}, \frac{23}{40}, \frac{9}{20}, \frac{9}{20}\right)$.

Notice that $\dot{y}^{P D}$ and $\dot{y}^{P I}$ are not core allocations. In particular, coalition $\{1,2,3\}$ pays $\frac{11}{10}$ with both rules, but can execute their jobs on their own at the cost of 1 . But notice that under our alternate interpretation of the game, coalition $\{1,2,3\}$ would have to pay their fair (proportional) share of the fixed cost if they secede, which is $\frac{3}{5}$, to which we add $\frac{\bar{c}^{J}(N)-1}{\bar{c}^{J}(N)}=\frac{1}{2}$ for the machine they need. We thus obtain $\tilde{C}\left(\{1,2,3\}, J, \frac{\bar{c}^{J}(N)-1}{\bar{c}^{J}(N)}\right)=\frac{7}{5}$. Thus $\dot{y}_{\{1,2,3\}}^{P D}=\dot{y}_{\{1,2,3\}}^{P I}=$ $\frac{11}{10}<\frac{7}{5}=\tilde{C}\left(\{1,2,3\}, J, \frac{\bar{c}^{J}(N)-1}{\bar{c}^{J}(N)}\right)$.

\section{Extension: general cost structure}

Up to this point, we have assumed that each additional machine entails the same marginal cost of 1 . In this subsection, we generalize this assumption by 
considering a vector $\theta \in \mathbb{R}_{+}^{\bar{c}^{J}(N)}$, where $\theta_{k}$ represents the marginal cost of the $k^{\text {th }}$ machine. A job scheduling problem with general cost function is thus a tuple $(N, J, \theta)$. In this context, the cost of a coalition $S$ is $\Theta(S, J, \theta) \equiv \sum_{k=1}^{\bar{c}^{J}(S)} \theta_{k}$.

Let $e=(1, \ldots, 1) \in \mathbb{R}_{+}^{\bar{c}^{J}(N)}$ be a vector of ones. The results of our previous sections hold essentially for problems of the form $(N, J, e)$.

In this extended framework, we say that a cost function $\theta$ exhibits decreasing average costs if

$$
\sum_{k=1}^{l} \frac{\theta_{k}}{l} \geq \sum_{k=1}^{\bar{c}^{J}(N)} \frac{\theta_{k}}{\bar{c}^{J}(N)}
$$

for all $1 \leq l \leq \bar{c}^{J}(N)$.

We show that the core is non-empty if and only if the cost function exhibits decreasing average costs. Define $\overline{\mathcal{Y}}(N, J, \theta) \equiv \mathcal{Y}(N, J, a(N, J, \theta) e)$, where $a(N, J, \theta)=\sum_{k=1}^{\bar{c}^{J}(N)} \frac{\theta_{k}}{\bar{c}^{J}(N)}$ is the average cost.

Theorem 6 Consider a problem $(N, J, \theta)$. Then $\operatorname{Core}(N, J, \theta) \neq \emptyset$ if and only if $\theta$ exhibits decreasing average costs. Moreover, if $\theta$ exhibits decreasing average costs, we have $\overline{\mathcal{Y}}(N, J, \theta) \subseteq \operatorname{Core}(N, J, \theta)$.

Proof. Necessity.

Notice first that for any $y \in \overline{\mathcal{Y}}(N, J, \theta), y(N)=\bar{c}^{J}(N) \sum_{k=1}^{\bar{c}^{J}(N)} \frac{\theta_{k}}{\bar{c}^{J}(N)}=\sum_{k=1}^{\bar{c}^{J}(N)} \theta_{k}=$ $\Theta(N, J, \theta)$.

Fix $S \subseteq N$. By definition of $\mathcal{Y}(N, J, a(N, J, \theta) e)$, we have for any $y \in$ $\overline{\mathcal{Y}}(N, J, \theta)$

$$
\begin{aligned}
y(S) & \leq \bar{c}^{J}(S) \sum_{k=1}^{\bar{c}^{J}(N)} \frac{\theta_{k}}{\bar{c}^{J}(N)} \\
& \leq \bar{c}^{J}(S) \sum_{k=1}^{\bar{c}^{J}(S)} \frac{\theta_{k}}{\bar{c}^{J}(S)} \\
& =\Theta(S, J, \theta)
\end{aligned}
$$

where the second inequality comes from decreasing average costs.

\section{Sufficiency.}

If $\theta$ does not have decreasing average costs then there exists $m<\bar{c}^{J}(N)$ for which $\sum_{k=1}^{m} \frac{\theta_{k}}{m}<\sum_{k=1}^{\bar{c}^{J}(N)} \frac{\theta_{k}}{\bar{c}^{J}(N)}$.

We have a partition $\left\{P^{1}, \ldots, P^{\bar{c}^{J}(N)}\right\}$ such that $\bar{c}^{J}\left(P^{k}\right)=1$ for all $k=$ $1, \ldots, \bar{c}^{J}(N)$.

Take a coalition $S$ consisting of $m$ elements of the partition $\left\{P^{1}, \ldots, P^{\bar{c}^{J}(N)}\right\}$. By definition, $\Theta(S, J, v)=\sum_{k=1}^{m} \theta_{k}$, yielding the core constraint $y(S) \leq \sum_{k=1}^{m} \theta_{k}$. Repeating the arguments for any subset of $m$ elements of the partition $\left\{P^{1}, \ldots, P^{\bar{c}^{J}(N)}\right\}$, 
we obtain the core constraints $y\left(P^{k}\right) \leq \sum_{k=1}^{m} \frac{\theta_{k}}{m}$, for all $k=1, \ldots, \bar{c}^{J}(N)$. Summing up, we obtain

$$
\begin{aligned}
\sum_{k=1}^{\bar{c}^{J}(N)} y\left(P^{k}\right) & =\sum_{i=1}^{n} y_{i} \\
& \leq \bar{c}^{J}(N) \sum_{k=1}^{m} \frac{\theta_{k}}{m} \\
& <\bar{c}^{J}(N) \sum_{k=1}^{\bar{c}^{J}(N)} \frac{\theta_{k}}{\bar{c}^{J}(N)} \\
& =\sum_{k=1}^{J} \theta_{k}=\Theta(N, J, \theta)
\end{aligned}
$$

where the first equality is because $\left\{P^{1}, \ldots, P^{\bar{c}^{J}(N)}\right\}$ is a partition of $N$ and the strict inequality is by our assumption that $\sum_{k=1}^{m} \frac{\theta_{k}}{m}<\sum_{k=1}^{\bar{c}^{J}(N)} \frac{\theta_{k}}{\bar{c}^{J}(N)}$.

Remark that one can easily adapt our two distinguished cost sharing rules (introduced in Subsection 3.2) to this extended framework. The peak-demand rule is defined in this context by $y_{i}^{P D}(N, J, \theta)=\frac{g_{i}^{P D}}{\bar{g}} a(N, J, \theta)$, for all $i \in N$.

Likewise, the peak-interval rule is defined in this context by $y_{i}^{P I}(N, J, \theta)=$ $\frac{\sum_{S \in G_{i}^{P D}} L(S)}{\sum_{T \in G^{P D}} L(T)} a(N, J, \theta)$, for all $i \in N$.

Corollary 2 On the set of job scheduling problems with general cost structure, the following results hold:

i) the peak-demand rule satisfies Strong Symmetry, No Merging No Splitting and Independence of Irrelevant Intervals;

ii) the peak-interval rule satisfies Strong Symmetry and Strong No Merging No Splitting;

iii) whenever Core $(N, J, \theta) \neq \emptyset$, we have $\hat{y}_{i}(N, J, \theta), \bar{y}_{i}(N, J, \theta) \in \operatorname{Core}(N, J, \theta)$.

Statements i) and ii) follow from the fact that properties therein are defined without referring to costs; and thus results are the same as in Section 3. Part iii) follows from Theorem 6 and the fact that both the peak-demand rule and the peak-interval rule always pick an allocation that is in $\mathcal{Y}(N, J, a(N, J, \theta) e)$.

\section{Concluding comments}

We have examined the cooperative game and the cost sharing problem associated with the classic job scheduling problem from operational research. It has been shown that only players who belong to peak-demand coalitions (which require the same number of machines as the grand coalition) may be charged 
a positive cost share under stable cost allocations. We have also studied two natural cost sharing rules that are core selections. The peak-demand rule is characterized by No Merging No Splitting, Independence of Irrelevant Intervals and Strong Symmetry. On the other hand, the peak-interval rule is characterized by Core Selection, No Merging No Splitting and Strong Symmetry.

It turns out that Core Selection is incompatible with Unanimity Lower Bound, a basic fairness requirement which requires that all agents pay an equal share of the first machine (which is needed by every player or coalition). On the other hand, the Shapley value satisfies Unanimity Lower Bound but not Core Selection. A compromise between these two axioms has been proposed by requiring stability for the modified problem where agents share the cost of the first machine equally.

We also studied the extension where the cost is not linear in the number of machines used, thus allowing for the possibility of decreasing or increasing returns to scale. In that context, the core of a job scheduling problem may be empty; and it has been shown that such a generalized job scheduling problem is balanced if and only if it exhibits decreasing average costs. Interestingly, we show that the respective properties of the peak-demand rule and the peakinterval rule are preserved in this extended model.

Another natural extension of the problem obtains by assuming that each agent may have multiple jobs to be executed. One possible way of allocating costs in this case is to consider each job of each agent as an individual player, share the cost as in our standard framework, and then charge to each agent the sum of the shares of her respective jobs. In particular, selecting a core allocation in the problem where each job is viewed as distinct player, and summing up the shares of the respective jobs of each owner will generate a core allocation in the extended problem with multiple jobs per agent. This approach would guarantee that the property No Merging No Splitting of Job Portfolio is met. As suggested by the name, this axiom says that agents should not benefit from splitting or merging their job portfolios.

Our work contributes to a growing body of literature that examines the cooperative aspects (and social choice implications) of classic problems from operational research. For example, the well-known minimum cost spanning tree problem has been studied (from a cost sharing perspective) by Bird (1976) and Bergantiños and Vidal-Puga (2007). Likewise, the minimum cost arborescence problem was introduced to this literature by Dutta and Mishra (2012) and also studied by Bahel and Trudeau (2017). Some similarities between these problems and the present one are (1) the existence of rules that are core selections and (2) the importance of using fairness criteria to choose between these rules. In comparison to the others, one remarkable aspect of the job scheduling problem is the simplicity of the algorithm allowing to find its optimal plan: this allows to define simple and intuitive rules. 


\section{References}

Bahel, E., Trudeau , C. (2017). Minimum Incoming Cost Rules for Arborescences. Social Choice and Welfare, 49(2): 287-314.

Bergantiños, G., Vidal-Puga, J. (2007). A fair rule in minimum cost spanning tree problems. Journal of Economic Theory, 137(1):326-352.

Bird, C. (1976). On cost allocation for a spanning tree: a game theoretic approach. Networks, 6:335-350.

Chun, Y., 1988. The proportional solution for rights problem. Mathematical Social Sciences 15, 231-246.

Chun, Y., 2016. Fair Queueing. Studies in Choice and Welfare, Springer, 157p.

Dantzig, G. B., Fulkerson, D. R., 1954. Minimizing the number of tankers to meet a fixed schedule. Naval Research Logistics Quarterly 1 (3), 217-222.

Dutta, B., Mishra, D. (2012). Minimum cost arborescences. Games and Economic Behavior, 74(1):120-143.

Elliyi, D., Azizgoglu, M., 2004. The fixed job scheduling problem: A literature review. Proceedings of the 24th National Congress on Operational Research and Industrial Engineering.

Gertsbakh, I., Stern, H. I., 1978. Minimal resources for fixed and variable job schedules. Operations Research 26 (1), 68-85.

Gupta, U. I., Lee, D. T., Leung, J. Y. T., 1979. An optimal solution for the channel-assignment problem. IEEE Trans. Comput. 28 (11), 807-810.

Hougaard, J., Moulin, H., 2014. Sharing the cost of redundant items. Games and Economic Behavior 87, 339-352.

Kar, A., 2002. Axiomatization of the Shapley Value on Minimum Cost Spanning Tree Games. Games and Economic Behavior 38, 265-277.

Kroon, L.G., Salomon, M., Van Wassenhove, L.N., 1995. Exact and approximation algorithms for the operational fixed interval scheduling problem. European Journal of Operational Research 82, 190-205.

Maniquet, F., 1996. Allocation Rules for a Commonly Owned Technology: The Average Cost Lower Bound. Journal of Economic Theory 69, 490-507.

Moulin, H., 1990. Joint Ownership of a Convex Technology: Comparison of Three Solutions. Review of Economic Studies 57, 439-452.

Myerson, R.B., 1977. Graphs and Cooperation in Games. Mathematics of Operations Research 2, 225-229. 
Núñez, M., Rafels, C., 2005. The böhm-bawerk horse market: a cooperative analysis. International Journal of Game Theory 33 (3), 421-430.

O'Neill, B., 1982. A problem of rights arbitration from the Talmud. Mathematical Social Sciences 2, 345-371.

Shapley, L. S., Shubik, M., 1971. The assignment game i: The core. International Journal of Game Theory 1 (1), 111-130.

Sprumont, Y., 2005. On the Discrete Version of the Aumann-Shapley CostSharing Method. Econometrica 73, 1693-1712.

Tejada, O., 2013. Analysis of the core of multisided böhm-bawerk assignment markets. TOP 21 (1), 189-205.

\section{A Appendix: Independence of properties}

\section{A.1 Theorem 2}

\section{A.1.1 No Merging No Splitting and Independence of Irrelevant In- tervals but not Strong Symmetry}

Let $S_{1} \in G^{P D}$ be such that $\max _{i \in S_{1}} s_{i}<\max _{i \in S_{j}} s_{i}$ for all $S_{j} \in G^{P D} \backslash S_{1}$. Let $w_{T}=1$ if $T=S_{1}$ and 0 otherwise. Let $y^{1}$ be the resulting allocation rule. We can verify that $y^{1}$ satisfies No Merging No Splitting and Independence of Irrelevant Intervals but not Strong Symmetry.

\section{A.1.2 No Merging No Splitting and Strong Symmetry but not In- dependence of Irrelevant Intervals}

The peak-interval rule satisfies No Merging No Splitting and Strong Symmetry but fails Independence of Irrelevant Intervals.

\section{A.1.3 Independence of Irrelevant Intervals and Strong Symmetry but not No Merging No Splitting}

Let $y_{i}^{E D}=\frac{\bar{c}^{J}(N)}{n}$ for all $i \in N$. We can verify that $y^{E D}$ satisfies Independence of Irrelevant Intervals and Strong Symmetry but not No Merging No Splitting.

\section{A.2 Theorem 3}

\section{A.2.1 Core Selection and Strong No Merging No Splitting but not Strong Symmetry}

We can verify that $y^{1}$ satisfies Core Selection and Strong No Merging No Splitting but not Strong Symmetry. 


\section{A.2.2 Core Selection and Strong Symmetry but not Strong No Merg- ing No Splitting}

The peak-demand rule satisfies Core Selection and Strong Symmetry but fails Strong No Merging No Splitting.

\section{A.2.3 Strong No Merging No Splitting and Strong Symmetry but not Core Selection}

Let $y_{i}^{P R O}=\frac{f_{i}-s_{i}}{\sum_{j \in N}\left(f_{j}-s_{j}\right)} \bar{c}^{J}(N)$ for all $i \in N$. We can verify that $y^{P R O}$ satisfies Strong Merging No Splitting and Strong Symmetry but not Core Selection.

\section{A.3 Theorem 4}

\section{A.3.1 Equal Treatment but not Full Incompatibility}

Let $y_{i}^{E D}=\frac{\bar{c}^{J}(N)}{n}$ for all $i \in N$. We can verify that $y^{E D}$ satisfies Equal Treatment but not Full Incompatibility.

\section{A.3.2 Full Incompatibility but not Equal Treatment}

The peak-demand rule satisfies Full Incompatibility but not Equal Treatment. To see this, suppose that $i \in N^{F}(J)$. Then, $i \in S$ for all $S \in G^{P D}(J)$, and thus $\bar{y}_{i}=1$.

In our running example, remember that $y^{P D}(N, J)=\left(0, \frac{1}{3}, \frac{2}{3}, \frac{2}{3}, \frac{1}{3}\right)$. Suppose that we modify $J^{\prime}$ so that $f_{1}^{\prime}=0.25$ and $f_{i}^{\prime}=f_{i}$ and $s_{i}^{\prime}=s_{i}$ otherwise. We have thus added an incompatibility between agents 1 and 2. Then, $G^{P D}\left(J^{\prime}\right)=$ $\{(1,2),(2,4),(3,4),(3,5)\}$. We thus have that $y^{P D}\left(N, J^{\prime}\right)=\left(\frac{1}{4}, \frac{1}{2}, \frac{1}{2}, \frac{1}{2}, \frac{1}{4}\right)$. The share of agent 1 has increase by $\frac{1}{4}$ but the share of agent 2 by $\frac{1}{6}$. 